\title{
AVALIAÇÃO DE PORTA-ENXERTOS DE VIDEIRA EM CONDIÇÕES SUBTROPICAIS $\left({ }^{1}\right)$
}

\author{
TANIA PIRES DA SILVA $\left({ }^{2}\right)$; RAFAEL PIO $\left(3^{*}\right)$; ARIANE BUSCH SALIBE $\left({ }^{4}\right)$; \\ IDIANA MARINA DALASTRA $\left({ }^{2}\right)$; JOSÉ RENATO STANGARLIN $\left({ }^{5}\right)$; ODAIR JOSÉ KUHN $\left({ }^{6}\right)$
}

\begin{abstract}
RESUMO
O objetivo deste trabalho foi avaliar porta-enxertos de videira na fase de formação em campo, bem como o desenvolvimento inicial do enxerto da variedade 'BRS Violeta'. Mudas oriundas de estacas enraizadas de 17 porta-enxertos de videira ('SO4', 'Teléki 8B', 'Harmony', 'Golia', 'Riparia de Traviú', 'Paulsen', '420 A', '99R', '5C', 'RR101-14', 'Kober 5BB', 'Rupestris Du Lot', 'IAC 313 Tropical', 'IAC 766 Campinas', 'IAC 572 Jales', 'VR 043-43' e 'IAC 571-6 Jundiaí') foram levadas ao campo no fim da primavera, em condições subtropicais. Após quatro meses, avaliou-se a severidade da antracnose e ferrugem da videira nas folhas das brotações dos porta-enxertos e após cinco meses o vigor dos mesmos. No inverno seguinte, foi realizada a enxertia por garfagem da variedade 'BRS Violeta' e após 60 dias, avaliaram-se a porcentagem de brotação, o diâmetro e o comprimento médio do enxerto. Nos porta-enxertos 'IAC 572 Jales' e 'IAC 313 Tropical' apresentaram menor infecção foliar a antracnose e ferrugem. O porta-enxerto 'IAC 572 Jales' proporcionou maior desempenho em campo, previamente à operação de enxertia, enquanto os porta-enxertos 'SO4', 'Harmony', 'Paulsen' e 'IAC 766 Campinas' promoveram maior vigor ao desenvolvimento do enxerto 'BRS Violeta'.
\end{abstract}

Palavras-chave: Elsinoe ampelina; Phakopsora euvitis; enxertia.

( $\left.{ }^{1}\right)$ Recebido para a publicação em 24 de novembro de 2008 e aceito em 20 de outubro de 2009.

$\left({ }^{2}\right)$ Universidade Estadual do Oeste do Paraná (Unioeste), Curso de Pós-graduação em Agronomia. Rua Pernambuco, 1777, Caixa Postal 1008, Centro, 85960-000 Marechal Cândido Rondon (PR). E-mail: taniapiresdasilva@yahoo.com.br; ididalastra@yahoo.com.br

$\left({ }^{3}\right)$ Universidade Federal de Lavras (UFLA), Departamento de Agricultura, Caixa Postal 3037, 37200-000 Lavras (MG). Bolsista Produtividade em Pesquisa CNPq. (*) Autor correspondente. E-mail: rafaelpio@dag.ufla.br

$\left({ }^{4}\right)$ Universidade Estadual do Oeste do Paraná (Unioeste), Bolsista de Desenvolvimento Cientifico e Tecnológico Regional Fundação Araucária. Rua Pernambuco, 1777, Caixa Postal 1008, Centro, 85960-000 Marechal Cândido Rondon (PR). E-mail: arianebs@unioeste.br

$\left({ }^{5}\right)$ Universidade Estadual do Oeste do Paraná (Unioeste), Rua Pernambuco, 1777, Caixa Postal 1008, Centro, 85960-000 Marechal Cândido Rondon (PR). Bolsista Produtividade em Pesquisa CNPq. E-mail: jrstangarlin@unioeste.br

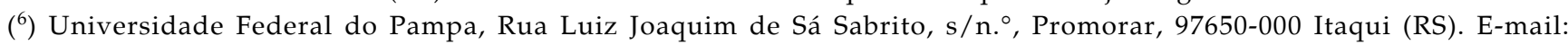
ojkuhm@gmail.com 


\title{
ABSTRACT \\ EVALUATION OF GRAPEVINE ROOTSTOCKS UNDER SUBTROPICAL CONDITIONS
}

\begin{abstract}
The work had as objective to evaluate the performance phytotechnical and disease tolerance of rootstocks of grapevines in the formation phase the field, as well as the initial development of graft variety "BRS Violeta". Seedlings originated from rooted cuttings of 17 rootstocks of grapevine ('SO4', 'Teléki 8B', 'Harmony', 'Golia', 'Riparia de Traviú', 'Paulsen', '420 A', '99R', '5C', 'RR101-14', 'Kober 5BB', 'Rupestris Du Lot', 'IAC 313 Tropical', 'IAC 766 Campinas', 'IAC 572 Jales', 'VR 043-43' and 'IAC 571-6 Jundiaí') were taken to the field at the end of spring in subtropical conditions. After four months, we evaluated the severity of anthracnose and rust of the grapevine on the leaves of the shoots of rootstocks and five months after the force of the same ones. The following winter, was performed cleft grafting the variety 'BRS Violeta' and after 60 days was evaluated for percentage of sprouting, the diameter and the average length of the graft. The rootstock 'IAC $572 \mathrm{Jales}^{\prime}$ and 'IAC 313 Tropical' had presented lower foliar infection anthracnose and rust. The rootstock 'IAC 572 Jales' showed higher performance in the field, prior to the operation of grafting, while the rootstock 'SO4', 'Harmony', 'Paulsen' and 'IAC 766 Campinas' promoted more vigorously the development of graft 'BRS Violeta'.
\end{abstract}

Key words: Elsinoe ampelina; Phakopsora euvitis; grafting.

No Oeste do Estado do Paraná, região tipicamente de clima subtropical, a viticultura foi introduzida recentemente e ainda está. Nesta região, os municípios de Toledo, Terra Roxa, Quatro Pontes e Marechal Cândido Rondon despontam como produtores de uvas americanas ou comuns (Vitis labrusca), para a produção de sucos e fermentados, com alguns pomares já instalados, mas ainda sem tecnologia gerada e adequada à região (WERLE et al., 2008).

Para a formação de um vinhedo, deve-se realizar, no campo, o plantio de estacas lenhosas sem raízes ou previamente enraizadas (barbados) dos porta-enxertos, efetuando-se a enxertia no inverno seguinte (Regina et al., 1998). A enxertia é uma das etapas críticas no processo de formação do vinhedo, cuja eficiência é dependente do acúmulo de reservas dos porta-enxertos (Pires e Biasi, 2003).

A diversificação de porta-enxertos pode ser uma estratégia importante em relação à ocorrência de doenças, como a antracnose, altamente influenciada pelos fatores climáticos de cada região, e observada nos cultivos comerciais de uva em diversos Estados brasileiros, (NAves et al., 2006). Outra doença que vem causando prejuízos sérios nos vinhedos é a ferrugem, que pode ocasionar redução do acúmulo de reservas, diante da desfolha precoce (SÔNEGO et al., 2005).

O objetivo do trabalho foi avaliar a tolerância a doenças foliares e o desempenho fitotécnico de diferentes porta-enxertos na fase de formação em campo nas condições subtropicais paranaenses.

Estacas caulinares lenhosas de 17 portaenxertos de videira ['SO4' - Vitis berlandieri $\times$ $V$. rupestris, 'Teléki $8 \mathrm{~B}^{\prime}-V$. berlandieri $\mathrm{x} V$. rupestris, 'Harmony' - $V$. candicans x $V$. champini, 'Golia' - $V$. riparia-carignane $\times V$. rupestris $d u$ lot, 'Riparia de
Traviú' $-V$. riparia $\times(V$. rupestris $\times V$. cordifolia $)$, 'Paulsen' - $V$. berlandieri $\times V$. rupestris, '420 A' - $V$. berlandieri $\times V$. riparia, '99R' $-V$. berlandieri $\times V$. rupestris, '5C'- $V$. berlandieri $\times V$. rupestris, 'RR101-14' - V.riparia $\times V$. rupestris, 'Kober $5 \mathrm{BB}^{\prime}-V$. berlandieri $\mathrm{x}$ V. riparia, 'Rupestris Du Lot' - V. rupestris, 'IAC 313 Tropical' - 'Golia' x V. cinerea, 'IAC 766 Campinas' 'Riparia de Traviú' x $V$. caribaea, 'IAC 572 Jales' - V. caribaea x '101-14 Mgt', 'VR 043-43' - V. vinifera $\times V$. rotundifolia e 'IAC 571-6 Jundiaí' - V. caribaea $\mathrm{x}$ 'Pirovano 57'] colhidas em julho de 2007 foram padronizadas com quatro gemas, comprimento de 20 $\mathrm{cm}$ e diâmetro ao redor de $12 \mathrm{~mm}$. Cada estaca foi plantada logo após a coleta em saco plástico de polietileno, tendo como substrato solo com textura argilosa. Em novembro de 2007, selecionaram-se 20 porta-enxertos (barbados) com maior número e comprimento das brotações de cada variedade para o transplante no campo. Passados 15 dias do plantio, selecionou-se uma única brotação, reduzindo-se a 20 $\mathrm{cm}$ de comprimento. O clima local é do tipo Cfa, subtropical com chuvas bem distribuídas e verões quentes. As temperaturas médias variaram entre 17,5 ${ }^{\circ} \mathrm{C}$ (mínima) e $28,5^{\circ} \mathrm{C}$ (máxima) (MAAK, 1981).

Após quatro meses do desenvolvimento dos porta-enxertos no campo, foi realizada a avaliação da severidade da doença antracnose da videira (Elsinoe ampelina) e 15 dias após para a ferrugem da videira (Phakopsora euvitis), ocorridas de forma natural. Para as avaliações de severidade das doenças, o delineamento adotado foi o inteiramente casualizado, com quatro repetições e cinco plantas por unidade experimental, avaliando-se três folhas em cada planta. Os tratamentos para a avaliação da severidade da antracnose constituíram-se dos seguintes porta-enxertos: 'SO4', 'Teléki 8B', 'Harmony', 'Golia', 'Riparia de Traviú', 
'Paulsen', '420 A', '99R', '5C', 'RR101-14', 'Kober 5BB', 'Rupestris Du Lot', 'IAC 313 Tropical', 'IAC 766 Campinas' e 'IAC 572 Jales'. Para a severidade da ferrugem foram avaliados os mesmos porta-enxertos, incluindo-se 'VR 043-43' e 'IAC 571-6 Jundiaí'.

A avaliação para a antracnose foi feita de acordo com sintomas descritos por Kimati e GalLI (1980) e para a ferrugem de acordo com os sintomas descritos por Leu (1988) e Tessmann e Vida (2005) e escada diagramática adaptada de AngelotTi et al. (2006). Utilizou-se escala de notas, variando de 1 a $10(1=$ sem lesão; $2=0,1 \%$ a $5 \% ; 3=5,1 \%$ a $10 \% ; 4=$ $10,1 \%$ a $15 \% ; 5=15,1 \%$ a $20 \% ; 6=20,1 \%$ a $25 \% ; 7=$ $25,1 \%$ a $30 \% ; 8=30,1 \%$ a $35 \% ; 9=35,1 \%$ a $40 \%$ e 10 $>40 \%$ ). O grau de tolerância proposto pela literatura, para a severidade da ferrugem, segundo a escala de notas é: até a nota $2=$ tolerante, de 2,1 a $4=$ moderadamente tolerante, de 4,1 a 7 = moderadamente suscetível e acima de 7 = suscetível; e para a severidade da antracnose: até a nota $2=$ tolerante, de 2,1 a 2,5 = moderadamente tolerante, de 2,6 a 3,0 = moderadamente suscetível e acima de 3,1 = suscetível.

Nove meses após o plantio dos porta-enxertos no campo em agosto de 2008, foi realizada a avaliação do vigor dos porta-enxertos. Os seguintes dados biométricos foram mensurados: número total de ramos, diâmetro médio dos ramos, comprimento do maior ramo e massa fresca total média dos ramos. $\mathrm{O}$ delineamento adotado foi em blocos ao acaso, com 17 tratamentos (porta-enxertos), quatro repetições e cinco plantas por unidade experimental.

Posteriormente às avaliações do vigor dos porta-enxertos, efetuou-se a enxertia pelo método de garfagem a $20 \mathrm{~cm}$ acima do solo, utilizando-se garfos parafinados e padronizados com $7,0 \mathrm{~cm}$ da variedade copa 'BRS Violeta'. O delineamento adotado foi em blocos ao acaso, com 17 tratamentos (porta-enxertos), quatro blocos e cinco enxertos por unidade experimental. Decorridos 60 dias da enxertia, mensurou-se a porcentagem de brotação, o diâmetro e o comprimento médio do enxerto.

Pela avaliação da severidade para a antracnose foliar verificou-se que os porta-enxertos 'IAC 572 Jales', 'IAC 766 Campinas' e 'IAC 313 Tropical' tiveram notas variando de 1,02 a 1,24, correspondendo à baixíssima porcentagem de área foliar lesionada, segundo a escala adotada para a avaliação.

Tabela 1. Severidade foliar de antracnose (Elsinoe ampelina) e ferrugem da videira (Phakopsora euvitis), número total de ramos (NR), diâmetro médio dos ramos (DR), comprimento do maior ramo (CR) e massa fresca total média dos ramos (MFR) de 17 porta-enxertos de videira em condições subtropicais, após nove meses do plantio, previamente à operação de enxertia

\begin{tabular}{|c|c|c|c|c|c|c|}
\hline Porta-enxertos & Antracnose & Ferrugem & NR & DR & CR & MFR \\
\hline & \multicolumn{2}{|c|}{$\longrightarrow$ pontos } & n. ${ }^{\circ}$ & $\mathrm{mm}$ & $\mathrm{cm}$ & $\mathrm{g}$ \\
\hline 'SO4' & $2,51 \mathrm{c}$ & $6,85 \mathrm{e}$ & $4,48 \mathrm{~b}$ & $7,53 \mathrm{~b}$ & $157,75 \mathrm{c}$ & $337,00 \mathrm{c}$ \\
\hline ‘Teléki 8B’ & $2,48 \mathrm{c}$ & $6,32 \mathrm{~d}$ & $2,81 \mathrm{~b}$ & $5,79 \mathrm{~b}$ & $103,25 \mathrm{c}$ & $263,75 \mathrm{~d}$ \\
\hline 'Harmony' & $3,06 \mathrm{~d}$ & $4,30 \mathrm{c}$ & $2,83 \mathrm{~b}$ & $8,86 \mathrm{a}$ & $114,50 \mathrm{c}$ & $428,25 \mathrm{~b}$ \\
\hline 'Golia' & $2,26 \mathrm{c}$ & $8,42 \mathrm{~g}$ & $4,32 \mathrm{~b}$ & 6,12 & $120,75 \mathrm{c}$ & $414,00 \mathrm{~b}$ \\
\hline 'IAC 313 Tropical' & $1,86 \mathrm{~b}$ & $7,34 \mathrm{f}$ & $3,75 \mathrm{~b}$ & $7,68 \mathrm{~b}$ & $281,25 \mathrm{~b}$ & $248,75 \mathrm{~d}$ \\
\hline$' 420 \mathrm{~A}^{\prime}$ & $2,94 \mathrm{~d}$ & 8,03 & $3,36 \mathrm{~b}$ & 6,17 & $120,00 \mathrm{c}$ & $476,25 \mathrm{~b}$ \\
\hline ‘Paulsen' & $2,81 \mathrm{~d}$ & $7,70 \mathrm{f}$ & $3,90 \mathrm{~b}$ & $8,37 \mathrm{a}$ & $113,00 \mathrm{c}$ & $459,75 \mathrm{~b}$ \\
\hline 'Riparia de Traviú' & $1,52 \mathrm{~b}$ & $8,52 \mathrm{~g}$ & $4,46 \mathrm{~b}$ & 6,10 & $111,75 \mathrm{c}$ & $367,75 \mathrm{c}$ \\
\hline 'RR101-14' & $1,75 \mathrm{~b}$ & $9,17 \mathrm{~h}$ & $5,60 \mathrm{a}$ & $6,73 \mathrm{~b}$ & $113,25 \mathrm{c}$ & $345,25 \mathrm{c}$ \\
\hline 'IAC 766 Campinas' & $2,15 c$ & $7,74 \mathrm{f}$ & $4,96 \mathrm{~b}$ & $8,25 \mathrm{a}$ & $253,75 \mathrm{~b}$ & $336,00 \mathrm{c}$ \\
\hline 'IAC 572 Jales' & $2,17 \mathrm{c}$ & $8,08 \mathrm{f}$ & $7,10 \mathrm{a}$ & $8,33 \mathrm{a}$ & $345,00 \mathrm{a}$ & $600,50 \mathrm{a}$ \\
\hline ‘99R' & $2,64 \mathrm{~d}$ & $7,54 \mathrm{f}$ & $6,16 \mathrm{a}$ & $6,97 \mathrm{~b}$ & $99,75 \mathrm{c}$ & $558,00 \mathrm{a}$ \\
\hline${ }^{\prime} 5 C^{\prime}$ & $1,24 \mathrm{a}$ & $1,39 \mathrm{a}$ & $4,25 \mathrm{~b}$ & $6,99 \mathrm{~b}$ & $111,50 \mathrm{c}$ & $437,50 \mathrm{~b}$ \\
\hline 'Rupestris Du Lot' & $1,09 \mathrm{a}$ & $3,04 \mathrm{~b}$ & $7,32 \mathrm{a}$ & $7,17 \mathrm{~b}$ & $105,50 \mathrm{c}$ & $400,93 \mathrm{c}$ \\
\hline 'Kober 5BB' & $1,02 \mathrm{a}$ & $1,49 \mathrm{a}$ & $4,53 \mathrm{~b}$ & $6,98 \mathrm{~b}$ & $129,75 \mathrm{c}$ & $454,37 \mathrm{~b}$ \\
\hline 'VR 043-43’ & - & $5,87 \mathrm{~d}$ & $5,83 \mathrm{a}$ & $9,52 \mathrm{a}$ & $146,50 \mathrm{c}$ & $344,37 \mathrm{c}$ \\
\hline 'IAC 571-6 Jundiaí' & - & $1,70 \mathrm{a}$ & $5,11 \mathrm{~b}$ & $9,60 \mathrm{a}$ & 180,66 c & $367,20 \mathrm{c}$ \\
\hline CV (\%) & 26,2 & 11,9 & 22,62 & 18,47 & 23,36 & 24,13 \\
\hline
\end{tabular}

Médias seguidas pela mesma letra na coluna não diferem entre si pelo teste de Scott-Knott $(P=0,05)$. 
Por outro lado, nos porta-enxertos 'Rupestris Du Lot', '420 A', 'Paulsen' e 'Harmony' observouse a maior porcentagem de área foliar lesionada, com notas variando de 2,64 a 3,06 (Tabela 1).

No geral, para a ferrugem foliar das videiras, constatou-se maior severidade a esta doença na grande maioria dos porta-enxertos avaliados. No entanto, nos

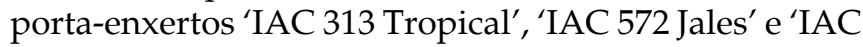
571-6 Jundiaí', notou-se a menor área foliar lesionada, com notas variando de 1,39 a 1,70, indicando potencial de tolerância a essa doença. Já em 'IAC 766 Campinas' a nota atribuída foi de 3,04 (Tabela 1). ANGelotti et al. (2008), avaliando as variedades 'IAC 313 Tropical', 'IAC 572 Jales' e 'IAC 766 Campinas' observaram necrose do tecido foliar ("flecks") ao redor das pústulas, o que indica a ocorrência de reação de hipersensibilidade por parte do hospedeiro e assim menor incidência de ferrugem nas folhas.

Quanto ao desempenho vegetativo, no portaenxerto 'IAC 572 Jales' notou-se maior comprimento do maior ramo ( $345 \mathrm{~cm})$, seguido do 'IAC 313 Tropical' $(281,25 \mathrm{~cm})$ e 'IAC 766 Campinas' $(253,75 \mathrm{~cm})$, que não diferiram entre si (Tabela 1). Para o diâmetro médio dos ramos, 'IAC 572 Jales' se destacou. Esses resultados concordam com BARROS et al. (1996), que avaliaram genótipos de videira para porta-enxerto e constataram que 'IAC 572 Jales' e 'IAC 766 Campinas', tiveram bom desenvolvimento vegetativo, previamente à enxertia. Nos porta-enxertos 'IAC 572 Jales', '99R', 'RR101-14', 'Rupestris Du Lot' e 'VR 043-43', observaram-se maior número de ramos (Tabela 1), mas somente para os dois primeiros houve também maior massa fresca total média dos ramos.

No presente estudo, maior sucesso dos enxertos da variedade 'BRS Violeta' na operação da enxertia foi obtida com o emprego dos porta-enxertos '420 A', 'SO4', 'Harmony', 'Paulsen' e 'IAC 766 Campinas', com índice de brotação do enxerto entre $70 \%$ e $77,5 \%$ (Tabela 2). No entanto, os últimos quatro apontados foram os que auxiliaram no maior sucesso no desenvolvimento do enxerto, pois, além de propiciarem elevado índice de brotação, contribuíram para o maior diâmetro e comprimento médio do enxerto. A importância do porta-enxerto ter bom desenvolvimento vegetativo previamente à operação de enxertia, principalmente no que se refere à produção de massa vegetal, está relacionada à síntese de fotoassimilados, que serão utilizados ao desenvolvimento radicular e acumulados durante o período de dormência. Após a operação da enxertia invernal em campo, maiores concentrações de carboidratos no sistema radicular poderão auxiliar no desenvolvimento vegetativo do enxerto, encurtando o tempo demandado para a formação do parreiral e ainda propiciando a formação de guias (braços) vigorosos.
Tabela 2. Porcentagem de brotação $(\mathrm{PB})$, diâmetro médio do enxerto (DE) e comprimento médio do enxerto (CE) da variedade 'BRS Violeta', em 17 porta-enxertos de videira, em condições subtropicais

\begin{tabular}{lccc}
\hline Porta-enxertos & PB & DE & CE \\
\hline & $\%$ & $\mathrm{~mm}$ & $\mathrm{~cm}$ \\
'SO4' & $75,00 \mathrm{a}$ & $6,69 \mathrm{a}$ & $45,07 \mathrm{a}$ \\
'Teléki 8B' & $45,00 \mathrm{c}$ & $3,26 \mathrm{~b}$ & $9,45 \mathrm{c}$ \\
'Harmony' & $75,00 \mathrm{a}$ & $5,77 \mathrm{a}$ & $44,45 \mathrm{a}$ \\
'Golia' & $35,00 \mathrm{~d}$ & $4,59 \mathrm{~b}$ & $21,93 \mathrm{~b}$ \\
'IAC 313 Tropical' & $62,50 \mathrm{~b}$ & $6,45 \mathrm{a}$ & $39,62 \mathrm{a}$ \\
'420 A' & $77,50 \mathrm{a}$ & $4,31 \mathrm{~b}$ & $26,35 \mathrm{~b}$ \\
'Paulsen' & $70,00 \mathrm{a}$ & $5,49 \mathrm{a}$ & $46,51 \mathrm{a}$ \\
'Riparia de Traviú' & $40,00 \mathrm{c}$ & $5,91 \mathrm{a}$ & $42,68 \mathrm{a}$ \\
'RR101-14' & $30,00 \mathrm{~d}$ & $4,32 \mathrm{~b}$ & $28,16 \mathrm{~b}$ \\
'IAC 766 Campinas' & $70,00 \mathrm{a}$ & $6,74 \mathrm{a}$ & $42,28 \mathrm{a}$ \\
'IAC 572 Jales' & $40,00 \mathrm{c}$ & $5,84 \mathrm{a}$ & $14,87 \mathrm{c}$ \\
'99R' & $35,00 \mathrm{~d}$ & $3,43 \mathrm{~b}$ & $14,91 \mathrm{c}$ \\
'5C' & $30,00 \mathrm{~d}$ & $2,77 \mathrm{~b}$ & $6,40 \mathrm{~d}$ \\
'Rupestris Du Lot' & $60,00 \mathrm{~b}$ & $7,13 \mathrm{a}$ & $38,00 \mathrm{a}$ \\
'Kober 5BB' & $30,00 \mathrm{~d}$ & $3,47 \mathrm{~b}$ & $21,12 \mathrm{~b}$ \\
'VR 043-43' & $15,00 \mathrm{e}$ & $0,98 \mathrm{~b}$ & $3,00 \mathrm{~d}$ \\
'IAC 571-6 Jundiaí' & $20,00 \mathrm{e}$ & $1,69 \mathrm{~b}$ & $4,50 \mathrm{~d}$ \\
\hline CV (\%) & 25,79 & 10,17 & 15,06 \\
\hline
\end{tabular}

Médias seguidas pela mesma letra na coluna não diferem entre si pelo teste de Scott-Knott $(\mathrm{P}=0,05)$.

Os porta-enxertos 'IAC 313 Tropical', 'Riparia de Traviú' e 'Rupestris Du Lot', também induziram bom desenvolvimento às brotações da copa, mas seu potencial de utilização pode ser comprometido pelo baixo índice de brotação na enxertia. Por outro lado, os dados obtidos para os porta-enxertos 'VR 043-43' e 'IAC 571-6 Jundiaí' indicam ser estes de comportamento pouco vigoroso, pois, além de baixa brotação na enxertia ( $15 \%$ e $20 \%$ respectivamente), promoveram desenvolvimento diminuto das brotações, com comprimento variando de 3,0 a $4,5 \mathrm{~cm}$ respectivamente (Tabela 2 ).

O resultado verificado para o porta-enxerto 'Paulsen' no bom desenvolvimento vegetativo da variedade 'BRS Violeta' sugere seu potencial de utilização em condições subtropicais. Este comportamento com a mesma variedade copa foi também observado em condições de clima temperado, nas serras gaúchas por CAMARGO et al. (2005), que constataram também bom desempenho da combinação em relação a produção de frutos. 
Para 'IAC 766 Campinas', não há informações na literatura sobre seu uso como porta-enxerto para a variedade 'BRS Violeta', mas aquele porta-enxerto foi descrito por TERRA et al. (2002), como o que induziu maior desenvolvimento vegetativo à copa 'Niágara Rosada' na região de Mococa (SP), por cinco anos consecutivos. A menor desfolha desse porta-enxerto possivelmente promoveu maior acúmulo de fotoassimilados, o que favoreceu a elevada porcentagem de brotação dos enxertos e seu alto desenvolvimento vegetativo. Ataques severos de antracnose e ferrugem em porta-enxertos no campo podem inviabilizar a formação do vinhedo, devido à desfolha precoce propiciar menor acúmulo de fotoassimilados e conseqüentemente diminuir significativamente o sucesso da operação da enxertia invernal, principalmente em porta-enxertos com menor vigor (FACHINELlo et al., 2005). Além do mais, a literatura cita 'IAC 766 Campinas' como um dos portaenxertos que possui maior potencial rizogênico, principalmente quanto à emissão de raízes (ТесCHIO et al., 2007).

De acordo com os resultados, concluiu-se que os porta-enxertos 'IAC 572 Jales', 'IAC 766 Campinas' e 'IAC 313 Tropical' possuem menor infecção foliar de antracnose, enquanto para a ferrugem os melhores resultados foram obtidos para as variedades 'IAC 313 Tropical', 'IAC 572 Jales' e 'IAC 571-6 Jundiaí'. No porta-enxerto 'IAC 572 Jales' constatou-se maior desenvolvimento, previamente à operação de enxertia, mas maior vigor das brotações de 'BRS Violeta' foi obtido quando enxertada sobre 'SO4', 'Harmony', ‘Paulsen' e 'IAC 766 Campinas'.

\section{AGRADECIMENTOS}

Os autores agradecem à Fundação Araucária de Apoio ao Desenvolvimento Científico e Tecnológico do Paraná pelo financiamento do projeto.

\section{REFERÊNCIAS}

ANGELOTTI, F.; TESSMANN, D.J.; SCAPIN, C.R.; VIDA, J.B.; OLIVEIRA, R.R. Elaboração e validação de escala diagramática para quantificação da severidade da ferrugem da videira. In: XXXIX CONGRESSO BRASILEIRO DE FITOPATOLOGIA, 39., 2006, Salvador Anais... Salvador: Fitopatologia Brasileira, 2006. p.31.

ANGELOTTI, F.; SCAPIN, C.R.; TESSMANN, D.J.; VIDA, J.B.; VIEIRA, R.A.; SOUTO, E.R. Resistência de genótipos de videira à ferrugem. Pesquisa Agropecuária Brasileira, v.43, p.11291134, 2008.
BARROS, J.C.S.M.; POMMER, C.V.; PASSOS, I.R.S.; TERRA, M.M.; SABINO, J.C.; RIBEIRO, I.J.A.; PIRES, E.J.P. Avaliação da capacidade de enraizamento e desenvolvimento vegetativo de genótipos de videira para porta-enxerto. Scientia Agricola, v.53, p.285-292, 1996.

CAMARGO, U.A.; MAIA, J.D.G.; NACHTIGAL, J.C. BRS Violeta: nova cultivar de uva para suco e vinho de mesa. Bento Gonçalves: Embrapa Uva e Vinho, 2005. 8p. (Embrapa Uva e Vinho. Comunicado Técnico 63)

FACHINELLO, J.C.; HOFFMANN, A.; NACHTIGAL, J.C. Propagação de plantas frutíferas. Brasília: Embrapa, 2005. 221p.

KIMATI, H.; GALLI, F. Doenças da Videira. In: GALLI, F. (Coord.). Manual de Fitopatologia: Doenças das plantas cultivadas. v.II. 2.ed. São Paulo: Agronômica Ceres, 1980. p.574-587.

LEU, L.S. Rust. In: PEARSON, R.C.; GOHEN, A.C. (Eds.). Compendium of grape diseases. St. Paul: APS Press, 1988. p.2830 .

MAACK, R. Geografia física do Estado do Paraná. 2.ed. Rio de Janeiro: J. Olympio, 1981. 450p.

NAVES, R.L.; GARRIDO, L.R.; SÔNEGO, O.R.; MÁRIO FOCHESATO, M. Antracnose da videira: sintomatologia, epidemiologia e controle. Bento Gonçalves: Embrapa Uva e Vinho, 2006. 32p. (Circular Técnica, 69)

PIRES, E.J.P.; BIASI, L.A. Propagação da videira. In: POMMER, C.V. (Ed.). Uva: tecnologia da produção, pós-colheita e mercado. Porto Alegre: Cinco Continentes, 2003. p.295-350.

REGINA, M.A.; SOUZA, C.R.; SILVA, T.G.; PEREIRA, A.F. A propagação da videira. Informe Agropecuário, v.19, p.20-27, 1998.

SÔNEGO, O.R.; GARRIDO, L.R.; GAVA, R. Ferrugem-davideira no Brasil. Bento Gonçalves: Embrapa Uva e Vinho, 2005. 4p. (Comunicado Técnico, 62)

TECCHIO, M.A.; MOURA, M.F.; HERNANDES, J.L.; PIO, R.; WYLER, P. Avaliação do enraizamento, desenvolvimento de raízes e da parte aérea de porta-enxertos de videira em condições de campo. Ciência e Agrotecnologia, v.31, p.18571861, 2007.

TERRA, M.M.; POMMER, C.V.; PIRES, E.J.P.; RIBEIRO, I.J.A.; GALLO, P.B. Production de trois variétés à raisins de table sur quatre porte-greffes au Brésil. Bulletin 1'O.I.V., v.75, p.4-20, 2002.

TESSMANN, D.J.; VIDA, J.B. A ferrugem-da-videira no Brasil. In: CONGRESSO BRASILEIRO DE FITOPATOLOGIA, 38., 2005, Brasília. Anais... Brasília: Sociedade Brasileira de Fitopatologia, 2005. p.220-222.

WERLE, T.; GUIMARÃES, V.F.; DALASTRA, I.M.; ECHER, M.M.; PIO, R. Influência da cianamida hidrogenada na brotação e produção da videira 'Niágara Rosada' na região Oeste do Paraná. Revista Brasileira de Fruticultura, v.30, p.20-24, 2008. 